\title{
Unfortunately, a "Back Light System" As a Global Positioning System Failed to Guide the Route in 25-G Fine-Needle Aspiration
}

\author{
Rungsun Rerknimitr ${ }^{1,2}$ and Phonthep Angsuwatcharakon ${ }^{2,3}$ \\ ${ }^{1}$ Division of Gastroenterology, Department of Medicine, Faculty of Medicine, ${ }^{2}$ Center of Excellence for Innovation and Endoscopy in \\ Gastrointestinal Oncology, Faculty of Medicine, ${ }^{3}$ Department of Anatomy, Faculty of Medicine, Chulalongkorn University and King \\ Chulalongkorn Memorial Hospital, Thai Red Cross Society, Bangkok, Thailand
}

See "A "Back Light System" for Identification of Sites for Endoscopic Ultrasound-Guided Fine-Needle Aspiration in Solid Pancreatic Masses: A Prospective, Randomized Study with a Crossover Design" by Ryo Harada, Hironari Kato, Soichiro Fushimi, et al., on page 334-339.

Driving a car these days is quite convenient using the global positioning system (GPS), which allows the driver access to many unfamiliar destinations without special training to drive in those routes. Performing endoscopic ultrasonography-guided fine-needle aspiration (EUS-FNA) with a rapid on-site evaluation (ROSE) by an attending cytopathologist is similar to driving with a human navigator, as this can reduce the errors of specimen inadequacy. ${ }^{1,2}$ Unfortunately, a human navigator is not always available in every road trip; similarly, ROSE is not always present in every endoscopy unit. The recently developed macroscopic on-site evaluation (MOSE) refers to an assessment of specimen adequacy using a visible whitish or yellowish core tissue while excluding paste- or liquid-like specimens. This assessment is performed by a trained EUS personnel. The specimen is processed separately, and the core tissue is micro-dissected and fixed with formalin, while the residual specimen is smeared on the glass slide and stained for cytological examination. MOSE is equivalent to ROSE in

Received: May 26, 2019 Revised: June 18, 2019

Accepted: July 23, 2019

Correspondence: Rungsun Rerknimitr

Division of Gastroenterology, Department of Medicine, Faculty of Medicine, Chulalongkorn University and King Chulalongkorn Memorial Hospital, Thai Red Cross Society, 1873 Rama IV Rd, Bangkok 10330, Thailand

Tel: +66-2-256-4265, Fax: +66-2-252-7839, E-mail: ERCP@live.com

ORCID: https://orcid.org/0000-0001-6866-6886

(c) This is an Open Access article distributed under the terms of the Creative Commons Attribution Non-Commercial License (http://creativecommons.org/ licenses/by-nc/3.0) which permits unrestricted non-commercial use, distribution, and reproduction in any medium, provided the original work is properly cited. terms of determining tissue adequacy and diagnostic accuracy, which are confirmed by cytopathologists. ${ }^{3}$ Bioevaluator ${ }^{\circledR}$ (Murazumi, Himeji, Japan) is based on a back light system (BLS) and is useful for evaluating specimens obtained using EUS-FNA, which may be obscured with blood, and for tissue extraction using forceps. Thus, it could be considered as the GPS for MOSE.

In this issue, a study group from Okayama University, Japan, evaluated specimen adequacy on the basis of 160 slides obtained from 80 patients with a pancreatic mass who underwent EUS-FNA with a 25-G needle for two passes. ${ }^{4}$ Tissue adequacy was assessed by a trained endosonographer who did not perform the EUS-FNA procedure. The endosonographer assessed specimen adequacy with or without using a BLS. ${ }^{4}$ The crossover design for using a BLS during the first and second passes was also applied. Only two-thirds of specimens in both groups were considered adequate $(52 / 80$ [65\%] in the BLS group vs. $54 / 80$ [68\%] in the non-BLS group, $p=0.88$ ). In addition, only fair agreement was observed between each endoscopist and the standard cytopathologist in both the BLS and non-BLS groups ( $k=0.40$ and 0.29 , respectively). As expected, the specimen obtained using a $25-\mathrm{G}$ needle, evaluated either with or without using a BLS, demonstrated suboptimal adequacy, as only two-thirds of the slides were considered adequate. Furthermore, the accuracy did not significantly differ ( $p=0.22)$ between with (76\%) and without BLS (66\%). The authors concluded that BLS did not improve the ability of specimen assessment. 
Although the authors admitted certain limitations in their study, such as the small sample size, specimens with less blood contamination, some necrotic or mucin-rich specimens, and the lack of experience of the endoscopists in using forceps for tissue extraction, we believe that the main pitfall is the use of a $25-$-G FNA needle, which yielded only $58 \%-86 \%$ of adequate specimens if only one pass was allowed, ${ }^{5}$ this seems to be inadequate as the European Society of Gastrointestinal Endoscopy guideline recommends that at least 3 or 4 passes should be performed to confirm the adequacy. ${ }^{6}$ Therefore, the advantage of BLS may not be demonstrated unless an FNA needle with a larger diameter is applied. In this study, the authors described that the reason for using a $25-\mathrm{G}$ needle was to ensure access to the lesion if the transduodenal approach was selected. In this situation, using a 19-G FNA needle definitely has significant difficulty, ${ }^{7}$ whereas FNA needles with a smaller diameter, such as 22 and $20 \mathrm{G}$, can still be used for transduodenal access and may yield larger specimens. ${ }^{5}$ Furthermore, needles with smaller sizes tend to have a higher discrepancy between the visible and histological cores. A study that used a $25-G$ needle reported that the visible core was recognized in $92 \%$ of the specimens, whereas the histological core was confirmed in only $32 \% .{ }^{8}$ The studies that used larger needles reported a lower discrepancy between the visible and histological cores of $91 \%$ and $79 \%$ with a $19-\mathrm{G}$ needle, ${ }^{3}$ and $96 \%$ and $81 \%$ with a $22-\mathrm{G}$ needle, ${ }^{9}$ respectively. A whitish specimen represents not only the pancreatic tissue but also the necrotic tissue or mucus. Within the smaller caliber of the $25-\mathrm{G}$ needle than that of larger FNA needles, the obtained tissue might easily mimic "core" tissue. In addition, the chance of having a bloody specimen is theoretically expected to be higher using larger FNA needles; ${ }^{10}$ perhaps, these bloody artifacts may potentially enhance the BLS distinction. In fact, this study ${ }^{4}$ allowed the third FNA pass with needles with larger diameters ranging from 22 to $19 \mathrm{G}$. Unfortunately, the results were not displayed or discussed. A study that used a $19-\mathrm{G}$ needle reported that a total length of $\geq 4 \mathrm{~mm}$ of visible core tissue was a good predictor for tissue accuracy and improved the diagnostic yield (area under the receiver-operating characteristic curve of 0.893). ${ }^{3}$ We suggest that the authors use third-pass specimens to test for specimen adequacy after testing with the BLS.

Nevertheless, we commend the idea of using the BLS for tissue adequacy distinction and encourage the team to expand their study to assess specimens obtained using larger needles. Perhaps, in driving through larger roads, the GPS may be of help.

\section{Conflicts of Interest}

The authors have no financial conflicts of interest.

\section{Acknowledgements}

This paper was supported by research grant for International Research Integration: Chula Research Scholar; Ratchadaphiseksomphot Endowment Fund, Chulalongkorn University, Bangkok, Thailand.

\section{REFERENCES}

1. Hébert-Magee S, Bae S, Varadarajulu S, et al. The presence of a cytopathologist increases the diagnostic accuracy of endoscopic ultrasound-guided fine needle aspiration cytology for pancreatic adenocarcinoma: a meta-analysis. Cytopathology 2013;24:159-171.

2. Matynia AP, Schmidt RL, Barraza G, Layfield LJ, Siddiqui AA, Adler DG. Impact of rapid on-site evaluation on the adequacy of endoscopic-ultrasound guided fine-needle aspiration of solid pancreatic lesions: a systematic review and meta-analysis. J Gastroenterol Hepatol 2014;29:697-705.

3. Iwashita T, Yasuda I, Mukai T, et al. Macroscopic on-site quality evaluation of biopsy specimens to improve the diagnostic accuracy during EUS-guided FNA using a 19-gauge needle for solid lesions: a single-center prospective pilot study (MOSE study). Gastrointest Endosc 2015;81:177-185.

4. Harada R, Kato H, Fushimi S, et al. A "back light system" for identification of sites for endoscopic ultrasound-guided fine-needle aspiration in solid pancreatic masses: a prospective, randomized study with a crossover design. Clin Endosc 2019;52:334-339.

5. Bang JY, Magee SH, Ramesh J, Trevino JM, Varadarajulu S. Randomized trial comparing fanning with standard technique for endoscopic ultrasound-guided fine-needle aspiration of solid pancreatic mass lesions. Endoscopy 2013;45:445-450.

6. Polkowski M, Jenssen C, Kaye P, et al. Technical aspects of endoscopic ultrasound (EUS)-guided sampling in gastroenterology: European Society of Gastrointestinal Endoscopy (ESGE) technical guideline - March 2017. Endoscopy 2017;49:989-1006.

7. Song TJ, Kim JH, Lee SS, et al. The prospective randomized, controlled trial of endoscopic ultrasound-guided fine-needle aspiration using $22 \mathrm{G}$ and 19G aspiration needles for solid pancreatic or peripancreatic masses. Am J Gastroenterol 2010;105:1739-1745.

8. Iwashita T, Nakai Y, Samarasena JB, et al. High single-pass diagnostic yield of a new 25-gauge core biopsy needle for EUS-guided FNA biopsy in solid pancreatic lesions. Gastrointest Endosc 2013;77:909-915.

9. Ishiwatari H, Sato J, Fujie S, et al. Gross visual inspection by endosonographers during endoscopic ultrasound-guided fine needle aspiration. Pancreatology 2019;19:191-195.

10. Gerke H. EUS-guided FNA: better samples with smaller needles? Gastrointest Endosc 2009;70:1098-1100. 